\title{
Das ambulante Borderline-Netzwerk in Berlin
}

\author{
Christian Stiglmayr
}

Online publiziert: 21 . Oktober 2020

(C) Der/die Autor(en) 2020

\begin{abstract}
Zusammenfassung Bei der Borderline-Persönlichkeitsstörung (BPS) handelt es sich um eine schwerwiegende psychische Störung mit weitreichenden Folgen für das Gesundheitssystem. Als Behandlungsmethode der Wahl wird Psychotherapie empfohlen, wobei die Dialektisch-Behaviorale Therapie (DBT) aufgrund ihrer vergleichsweise vielen Wirksamkeitsnachweise in den S2-Leitlinien für Persönlichkeitsstörungen deutscher Fachgesellschaften mit dem höchsten Evidenzgrad geführt wird. Trotzdem ist es für Patient_innen mit BPS schwierig, adäquate ambulante Hilfe zu erhalten. Aus diesem Grund haben sich in Städten und Gemeinden spezifische Netzwerke gegründet, mit dem Ziel eines verbesserten Versorgungsangebots für Patient_innen mit BPS. Der Artikel beschreibt exemplarisch das 2002 gegründete Berliner Borderline-Netzwerk, welches schwerpunktmäßig mit DBT arbeitet. Von 2007 bis 2010 fand eine umfangreiche Evaluation dieses Netzwerkes statt, die Ergebnisse werden zusammenfassend präsentiert. Der Artikel schließt mit einer abschließenden Würdigung der Vor- und Nachteile eines solchen Netzwerkes sowie einem Ausblick, was für eine noch bessere Versorgung von Patient_innen mit BPS wünschenswert ist.
\end{abstract}

Schlüsselwörter Borderline-Persönlichkeitsstörung (BPS) · Dialektisch-Behaviorale Therapie (DBT) · Borderline-Netzwerk Berlin · Ambulante Versorgung • Berliner Borderline-Versorgungsstudie (BBV)

C. Stiglmayr $(\bowtie)$

Arbeitsgemeinschaft für Wissenschaftliche Psychotherapie Berlin (AWP Berlin), Tempelhofer Damm 227, 12099 Berlin, Deutschland

christian_stiglmayr@web.de

\section{The outpatient DBT network in Berlin}

Summary Borderline personality disorder (BPD) is a serious mental disorder with far-reaching consequences for the health system. Psychotherapy is recommended as the treatment method of choice, with Dialectical-Behavioral Therapy (DBT) being listed with the highest level of evidence in the S2 guidelines for personality disorders of German specialist societies due to its comparatively most evidence of effectiveness. Even so, it is difficult for patients with BPD to get adequate outpatient help. For this reason, specific networks have been set up in cities and municipalities with the aim of improving the range of care for patients with BPD. The article describes the Berlin DBT network founded in 2002 as an example. An extensive evaluation of this network took place from 2007 to 2010 and the results are presented in summary. The article closes with a final appreciation of the advantages and disadvantages of such a network as well as an outlook on what is desirable for even better care of patients with BPD.

Keywords Borderline personality disorder (BPD) Dialectical-Behavioral Therapy (DBT) · Borderline-

Network Berlin · Outpatient care · The Berlin Borderline outpatient care-study (BBV)

\section{Einführung}

Bei der Borderline-Persönlichkeitsstörung (BPS) handelt es sich um eine schwerwiegende psychische Störung mit weitreichenden Folgen für das Gesundheitssystem. Als Kernmerkmal der BPS gilt ein durchgängiges Muster von Instabilität im Bereich der Affekte i. S. einer „Affektlabilität“. Die Affekte können sich innerhalb von Sekunden verändern, was von den Betroffenen als nur schwer kontrollierbar erlebt wird. 
Weitere zentrale Merkmale der BPS sind eine Störung der Identität sowie eine Störung der zwischenmenschlichen Beziehungsgestaltung. Selbstverletzendes Verhalten ist bei ca. $70 \%$, Suizidversuche bei ca. $60 \%$ der Betroffenen zu beobachten. In 7 bis $10 \%$ der Fälle kommt es zu einem vollendeten Suizid. Alkoholund Drogenmissbrauch, Hochrisikoverhalten und andere schwerwiegende dysfunktionale Verhaltensweisen sind häufig. Gelegentlich fallen Borderline-Betroffene durch ausgesprochen aggressive Verhaltensweisen auf, häufiger ist allerdings, dass sie diese Aggressionen gegen sich selbst richten.

Borderline-Patient_innen sind ausgesprochen gequälte Menschen, bis zu $80 \%$ suchen aus diesem Grund Hilfe im Gesundheitssystem auf (Lieb et al. 2004) und beanspruchen das Gesundheitssystem damit in einem größeren Umfang als zum Beispiel Patient_innen mit einer Major Depression (Bender et al. 2001; Zanarini et al. 2004). Wird eine Prävalenzrate von ca. 1 bis $1,5 \%$ der erwachsenen Bevölkerung zugrunde gelegt (Coid, et al. 2006; Lenzenweger et al. 2007), bedeutet dies für Deutschland eine Zahl von 500.000 bis $\mathrm{zu} 700.000$ behandlungsbedürftigen erwachsenen Borderline-Patient_innen.

Allerdings wird Patient_innen mit einer BPS häufig mit ausgeprägten Vorurteilen begegnet, z.B. sie seien manipulativ, unberechenbar oder grenzüberschreitend. Bis zu einem Viertel der ambulant tätigen Therapeut_innen lehnt aus diesem Grund eine Zusammenarbeit ab (Jobst et al. 2009), andere lassen sich auf eine Behandlung zwar ein, sind aber angesichts der Vielzahl an Problemen häufig überfordert. Die Folge ist, dass die Betroffenen stationäre Einrichtungen aufsuchen, die nur in wenigen Fällen eine spezifische psychotherapeutische Behandlung vorhalten. Dies führt nicht selten dazu, dass BorderlinePatient_innen mehrmals im Jahr stationär aufgenommen werden müssen. Dies führt nicht nur zu einer erheblichen Belastung der Patient_innen sondern auch des Gesundheitssystems; die Krankheitskosten betragen pro Patient pro Jahr durchschnittlich $27.000 €$. Diese Kosten werden nur noch von den Krankheitskosten von Patient_innen mit einer Schizophrenie und einer Multiplen Sklerose übertroffen (Wagner et al. 2013).

\section{Die Dialektisch-Behaviorale Therapie (DBT)}

Als Behandlungsmethode der Wahl wird Psychotherapie empfohlen, wobei die Dialektisch-Behaviorale Therapie (DBT) aufgrund ihrer vergleichsweise vielen Wirksamkeitsnachweise in den S2-Leitlinien für Persönlichkeitsstörungen deutscher Fachgesellschaften (DGPPN 2009) mit dem höchsten Evidenzgrad geführt wird. Die DBT wurde in den 1980er-Jahren als manualgestützte verhaltenstherapeutisch orientierte Therapie von Marsha Linehan entwickelt (Linehan 1996a, 1996b). Neben den etablierten behavioralen Methoden integriert die DBT eine Vielzahl weite- rer Strategien und Techniken, vor allem aus der Gesprächspsychotherapie, der Gestalttherapie, der Hypnotherapie und dem Zen-Buddhismus. Im Verständnis der DBT wenden die Betroffenen die meisten dysfunktionalen Verhaltensweisen wie z.B. schweres selbstverletzendes Verhalten oder Suizidversuche an, um unerträgliche affektive Zustände $\mathrm{zu}$ regulieren. Primäres Ziel der DBT-Behandlung ist demnach der Aufbau bzw. die Verbesserung von funktionalen bei gleichzeitigem Abbau von dysfunktionalen Verhaltensweisen zur Affektregulation. Übergeordnetes Ziel der DBT ist die Auflösung dichotomer Betrachtungsweisen von sich und seiner Umgebung und damit ein tiefes inneres Annehmen von dem, was ist.

Die ambulante Therapie ist auf ein bis drei Jahre angelegt. Der Ablauf der Therapie ist klar strukturiert und lässt sich in drei Behandlungsstadien unterteilen. Das erste Therapiestadium dient der Verbesserung von Verhaltenskontrolle bei bestehendem schwerwiegenden Problemverhalten. Wesentlicher Bestandteil dieses ersten Therapiestadiums ist ein gemeinsam unterzeichneter Therapievertrag. Dieser beinhaltet die wichtigsten Therapieziele und die Einhaltung bestimmter Therapievereinbarungen. Unter anderem verpflichtet sich die Patient_in, während der gesamten Therapiedauer keinen Suizidversuch zu begehen. Die Therapievereinbarungen gelten zunächst für die Dauer eines Jahres (ggf. auch kürzer). Die Fortsetzung der Behandlung wird von einem erfolgreichen Verlauf der Therapie abhängig gemacht. Nach der Unterzeichnung des Therapievertrages besucht die Patient_in zusätzlich ein Fertigkeitentraining. Zentral für das erste Therapiestadium ist die Reduktion und Unterbindung bislang dysfunktionaler Verhaltensweisen zur Emotionsregulation bei gleichzeitiger Vermittlung alternativer, funktionaler Strategien. Damit wird der Weg frei für das zweite Therapiestadium, in welchem es vorrangig um die Bearbeitung von Problemen des emotionalen Erlebens sowie der Erhöhung der emotionalen Belastbarkeit geht. Bei Bestehen einer komorbiden komplexen Posttraumatischen Belastungsstörung ist auch deren Behandlung Bestandteil dieses Therapiestadiums. Das abschließende dritte Therapiestadium dient der Gestaltung eines sinnerfüllten Lebens sowie des tiefen inneren Annehmens der eigenen Person. Hier kommen insbesondere Techniken und Haltungen des achtsamen Selbstmitgefühls zum Einsatz (Germer 2012).

Allen drei Behandlungsstadien gemein ist eine sehr klare Struktur, welche durch eine dynamische Behandlungshierarchie vorgegeben wird: wann immer lebensgefährliches Verhalten auftritt, muss dieses vorrangig behandelt werden, gefolgt von Verhalten, welches $\mathrm{zu}$ einem Therapieabbruch bzw. Verhalten, welches zu schweren Krisen führen könnte; tritt keines dieser Verhaltensweisen auf, besteht für die Therapiestunde freie Problemwahl. 


\section{DBT-Therapiebausteine}

Die DBT besteht aus einer Einzeltherapie, einem Fertigkeitentraining in der Gruppe, Telefonkontakten zur Krisenintervention, einem Konsultationsteam sowie einer bedarfsorientierten Supervision.

\section{DBT-Einzeltherapie}

Die Einzeltherapie findet regelhaft einmal die Woche statt. Hauptaufgabe der Einzeltherapie ist neben der individuellen Ziel- und Motivationsarbeit die Durchführung von Problem- und Lösungsanalysen. Gleichzeitig zeichnet sich die Einzeltherapie durch einen starken Emotionsfokus aus, wobei zu Beginn der Therapie vor allem die Kontrolle, später das Zulassen und Akzeptieren der Emotionen vorrangig ist.

\section{DBT-Fertigkeitentraining}

Das parallel verlaufende Fertigkeitentraining findet ebenfalls einmal in der Woche statt, dauert jeweils $11 / 2$ bis zwei Zeitstunden und wird in einer Gruppe von maximal sieben bis acht Patient_innen durchgeführt. Das Training wird von zwei Trainer_innen geleitet. Alleiniges Ziel des Fertigkeitentrainings ist die Vermittlung von spezifischen Fertigkeiten, welche für ein therapeutisches Fortkommen als unverzichtbar angesehen werden. Die Fertigkeiten stellen den Ersatz für bislang dysfunktionales Verhalten dar, bilden aber auch die Grundlage für die persönliche Weiterentwicklung im Sinne der in Therapiephase 3 definierten Ziele. Es ist empfehlenswert, wenn die Einzeltherapie sowie das Fertigkeitentraining nicht in Personalunion stattfinden, da das Fertigkeitentraining ausschließlich der Vermittlung von Fertigkeiten dient und damit einen schulischen und ausdrücklich nicht psychotherapeutischen Fokus hat. Die Inhalte des Fertigkeitentrainings sind in dem Buch von Bohus und Wolf (2013) umfangreich dargestellt.

Sollte, wie das in vielen ländlichen Regionen der Fall ist, keine Fertigkeitengruppe zur Verfügung stehen, müssen die Fertigkeiten in der Einzeltherapie vermittelt werden. Dabei hat es sich bewährt, für die Vermittlung der Fertigkeiten einen separaten Termin $\mathrm{zu}$ vereinbaren, z.B. alle zwei bis drei Wochen eine zusätzliche Stunde in der Woche. Dieses Vorgehen erleichtert die Trennung von Prozessen, die in der Einzeltherapie fokussiert werden müssen und der eher pädagogisch orientierten Fertigkeitenvermittlung.

\section{Telefonkontakte}

Telefonkontakte werden zum Management von akuten Krisen angeboten. Die Patient_in hat die Möglichkeit, im Rahmen von vorher klar definierten Zeiträumen die Therapeut_in anzurufen (z. B. zwischen 9 und 19 Uhr, außer am Wochenende; Rückrufgarantie innerhalb von $24 \mathrm{~h}$, außer am Wochenende). Diese vermittelt der Patient_in effektive Fertigkeiten, um mit der aktuellen Situation umgehen zu können. Das Gespräch sollte nicht länger als 3 bis 5 min dauern; es wird explizit keine Therapie am Telefon durchgeführt. Vorrangige Aufgabe der Telefonkontakte ist damit der Transfer des in der Therapie Erlernten in den Alltag der Patient_in.

\section{Konsultationsteam}

Um die Belastung der Therapeut_in zu reduzieren und die Effektivität der Behandlung zu steigern, wird die DBT im Team durchgeführt. Ein sogenanntes Konsultationsteam trifft sich wöchentlich bis vierzehntägig und besteht aus Einzeltherapeut_innen und Fertigkeitentrainer_innen, ggf. auch weiteren an der Behandlung beteiligten Personen. Dort werden nach festen Regeln die Patient_innen einer jeden Einzeltherapeut_in besprochen und das weitere therapeutische Vorgehen festgelegt. Aufgabe des Konsultationsteams ist demnach zweierlei: zum einen hat das Team dafür Sorge zu tragen, dass die Patient_in die bestmögliche DBT erhält. Zum anderen ist es Aufgabe des Teams, die Behandlung gemeinsam durchzuführen. Sämtliche therapeutische Schritte werden im Team beschlossen und sind anschließend von der zuständigen Einzeltherapeut_in umzusetzen. Im Unterschied zu den meisten anderen Therapien wird die Behandlung demnach nicht von einer Einzelperson sondern von einem Therapeutenteam durchgeführt.

Damit ist das Konsultationsteam das "Herzstück“ der DBT. Hier laufen alle Informationen zusammen und werden ausgewertet. Darauf aufbauend wird beschlossen, was bis zum nächsten KonsultationsteamTreffen von den Einzeltherapeut_innen umgesetzt werden muss. Anschließend gehen alle Einzeltherapeut_innen auseinander um die Teambeschlüsse zu realisieren. Zum nächsten Konsultationsteam kommen alle Therapeut_innen wieder zusammen um von den Ergebnissen ihrer Arbeit zu berichten. Dieser Zyklus stellt eine stabile Grundordnung innerhalb der DBT dar; das Konsultationsteam ist Motor und Ruhepol zugleich.

Es nehmen in der Regel zwischen 3 und 5 Therapeut_innen an dem Konsultationsteam teil. Zumeist schließen sich diejenigen zusammen, die in demselben Ortsteil wohnen und/oder die sich besonders zugetan sind.

Sollten die Fertigkeitentrainer_innen nicht am Konsultationsteam teilnehmen können, hat es sich bewährt, wenn sie im Anschluss an jedes Fertigeitentraining eine Rundmail an die zuständigen Einzeltherapeut_innen mit den wichtigsten Informationen verschicken.

\section{Supervision}

In der zusätzlichen Supervision können individuelle Prozesse und Schwierigkeiten besprochen werden, die im Rahmen des Konsultationsteams nicht aufgefangen und bearbeitet werden können. Supervision ist auch immer dann notwendig, wenn es zu (interaktionellen) Problemen innerhalb des Konsultationsteams kommt. 


\section{Die Entwicklung von Borderline-Netzwerken}

Zur Verbesserung des Versorgungsangebots für Patient_innen mit BPS haben sich in vielen Städten und Gemeinden in Deutschland, aber auch der Schweiz und Österreich, Netzwerke gegründet. Häufig ist die Motivation zur Gründung eines Borderline-Netzwerkes aber auch, den Teamgedanken der DBT besser umsetzen zu können. Den Netzwerken können sowohl ambulant, (teil-)stationär wie auch komplementär arbeitende Personen angehören. Rein ambulante Borderline-Netzwerke können im Rahmen von Institutsambulanzen, Psychotherapie-Ausbildungsinstituten oder als Netzwerke von Niedergelassenen stattfinden. Das kleinstmögliche Borderline-Netzwerk besteht aus zwei sich regelmäßig treffenden Therapeut_innen; das kleinste DBT-Netzwerk besteht aus drei Therapeut_innen, einer Einzeltherapeut_in und zwei Fertigkeitentrainer_innen. Es gibt unspezifisch wie auch spezifisch ausgerichtete Borderline-Netzwerke. Therapeut_innen, die in unspezifischen Borderline-Netzwerken mitwirken, arbeiten mit unterschiedlichen psychotherapeutischen Ausrichtungen (z.B. Verhaltenstherapie, Tiefenpsychologisch Fundierte Psychotherapie, Psychoanalyse, Systemische Therapie, Gesprächspsychotherapie). Spezifischen Borderline-Netzwerke gehören hingegen meist Therapeut_innen an, die sich den Grundprinzipien einer bestimmten Behandlungsmethode verpflichten. Die meisten spezifischen Borderline-Netzwerke arbeiten nach den Grundprinzipien der DBT. Größere spezifisch sowie unspezifisch ausgerichtete Netzwerke bestehen aus bis zu 30 Therapeut_innen (z. B. Hamburg und Berlin). Die in den Netzwerken vertretenen Berufsbilder sind zumeist ärztliche und psychologische Psychotherapeut_innen. Aber auch andere Berufsbilder sind denkbar; beispielsweise werden viele DBT-Fertigkeitengruppen von Ergotherapeut_innen angeboten.

\section{Das Berliner Borderline-Netzwerk}

Das Berliner Borderline-Netzwerk wurde 2002 mit den Zielen einer besseren Versorgung von Patient_innen mit einer BPS wie auch der Unterstützung der mit Borderliner_innen arbeitenden Therapeut_innen gegründet. Zu Beginn bestand das Netzwerk aus fünf niedergelassenen sowie zwei stationär arbeitenden ärztlichen und psychologischen Psychotherapeut_innen, die allesamt in DBT fortgebildet waren. Monatliche Treffen dienten dem Austausch über einzelne Patient_innen, aber auch der gegenseitigen Unterstützung. Konsultationstreffen wurden erst zu einem späteren Zeitpunkt etabliert (2007 mit Beginn einer Studie zur Effektivität der angebotenen DBT; siehe Kap. „Die Berliner Borderline-Versorgungsstudie (BBV)“). Der Kerngruppe schlossen sich über die Jahre immer weitere Kolleg_innen an, so dass das Netzwerk rasch auf bis zu 40 Personen anwuchs. Gute Erfah- rungen wurden damit gemacht, gezielt Kolleg_innen anzusprechen, die aus anderen Kontexten bekannt waren (z. B. gemeinsame Klinikzeit, Zusammenarbeit in Qualitätszirkeln und/oder Intervisionsgruppen). Da zur Teamarbeit eine hohe Bereitschaft gehört, sich auch mit seinen Fehlern zu zeigen, hat es sich bewährt, vor allem Kolleg_innen ansprechen, zu denen ein Vertrauensverhältnis besteht und mit denen eine Zusammenarbeit einen Mehrwert darstellt. Zum Aufbau eines ambulanten Netzes gehört sicherlich auch der Spaß an Teamarbeit. Hilfreich war auch, dass sich mehrere (teil-) stationäre wie auch komplementäre Einrichtungen in DBT fortbildeten und sich teilweise auch zertifizieren ließen (DDBT e.V.).

Es wurde eine eigene Homepage entwickelt, welche u. a. eine Therapeut_innenliste, eine Liste angebotener DBT-Fertigkeitengruppen und eine Liste an stationären und teilstationären Einrichtungen beinhaltet (www.borderline-netzwerk-berlin.de). Im Rahmen des Berliner Borderline-Netzwerkes werden durchschnittlich 30 Therapeut_innen, 8 Fertigkeitengruppen, 5 komplementäre sowie 8 vollstationäre bzw. teilstationäre Einrichtungen vorgehalten. Mit Ausnahme weniger ambulanter Therapeut_innen wird zur Behandlung der Borderline-Patient_innen ausschließlich DBT angeboten.

Der Zusammenhalt eines solchen Netzwerkes kann durch die Organisation regionaler (DBT-) Fortbildungen, Infoveranstaltungen und Kongressen oder die gemeinsame Entwicklung einer Studie zur Evaluierung des Netzwerkes (so geschehen in Berlin und Darmstadt; Stiglmayr et al. 2015; Friedrich et al. 2003) befördert werden.

\section{Finanzierung}

Die DBT zählt zu den verhaltenstherapeutischen Methoden und kann in Deutschland im Rahmen des Richtlinienverfahrens Verhaltenstherapie mit der Krankenkasse abgerechnet werden. Auch das dazugehörige Fertigkeitentrainng wird bei entsprechender Eignung der Fertigkeitentrainer_innen (Gruppenzulassung) von der Krankenkasse übernommen. Allerdings werden Einzeltherapie und Fertigkeitentraing in diesem Fall gemeinsam veranschlagt. Aus diesem Grund ist eine solche Umsetzung häufig nicht praktikabel, da ansonsten $\mathrm{zu}$ wenige Stunden für die Einzeltherapie verbleiben. Nachfolgend werden die häufigsten Finanzierungsmöglichkeiten benannt, ohne auf diese hier näher einzugehen. Konkretere Auskünfte erhalten die Patient_innen von den Fertigkeitentrainer_innen oder jeweils zuständigen Institutionen:

- Die Patientin trägt die Kosten für das Fertigkeitentraining selbst

- Die Kosten werden im Rahmen des persönlichen Budgets übernommen (SGB IX) 
- Die Kosten werden in einer Sonderregelung zusätzlich zur Einzeltherapie von der Krankenkasse übernommen

- Das Fertigkeitentraining wird im Rahmen einer ergotherapeutischen Leistung angeboten und entsprechend abgerechnet

- Das Fertigkeitentraining wird im Rahmen einer psychiatrischen Institutsambulanz (PIA) angeboten

Übernehmen die Patient_innen die Kosten selbst, fallen je nach Finanzierungsmodell der Ferigkeitentrainer_innen zwischen 5 und $60 €$ pro Termin an.

\section{Die Berliner Borderline-Versorgungsstudie (BBV)}

Im Rahmen des Netzwerkes entstand schließlich die Frage nach der Effektivität der psychotherapeutischen Angebote. Spezifisch interessierte die Frage, ob die im Rahmen des Netzwerkes angebotene DBT („DBT as usual“) mit der DBT im Rahmen von publizierten RCTs hinsichtlich Inhalt und Effektivität vergleichbar ist. Die Studie wurde in den Jahren 2007 bis 2010 durchgeführt und fand in Kooperation zwischen der Freien Universität Berlin (FU), der Humboldt Universität zu Berlin (HU), der Charité sowie der Arbeitsgemeinschaft für Wissenschaftliche Psychotherapie Berlin (AWP Berlin) statt. Der Untersuchungszeitraum betraf das erste Jahr DBT, eine Follow-Up-Messung fand nach einem weiteren Jahr statt. Eingeschlossen wurden Patientinnen und Patienten mit einer diagnostizierten Borderline-Persönlichkeitsstörung nach DSMIV-R (American Psychiatric Association 2003) im Alter ab dem 16 Lebensjahr; um mit den einschlägigen Studien vergleichbar $\mathrm{zu}$ sein waren die Ausschlusskriterien eine Schizophrenie (lifetime), eine Bipolare Störung (liefetime), eine akute Abhängigkeitsstörung, eine Antisoziale Persönlichkeitsstörung, eine auffällige Intelligenzminderung, eine akute Suizidalität sowie eine laufende Psychotherapie.

Die Rekrutierung der Patient_innen erfolgte über das bestehende Berliner Borderline-Netzwerk einschließlich der dazugehörigen Homepage. Nach ei- nem Telefonscreening wurde zur Prüfung der Einund Ausschlusskriterien das Strukturierte Interview für Klinische Störungen Achse I (SKID I; Wittchen et al. 1997) und Achse II (SKID II; Fydrich et al. 1997) durchgeführt.

Insgesamt wurden 297 Personen (259 Frauen; 38 Männer) am Telefon einem ersten Screening unterzogen, wovon schließlich 78 Patient_innen in die Studie eingeschlossen wurden. Die Therapie begonnen haben 70 Patient_innen (11 Männer, 59 Frauen), 47 (4 Männer, 43 Frauen) befanden sich nach einem Jahr noch in Therapie (es bestand die Möglichkeit, die DBT über das Erhebungsjahr hinaus aufzusuchen). Die Abbrecherquote betrug damit 32,9\%.

Insgesamt nahmen 29 Therapeut_innen aus dem Netzwerk (7 Männer, 22 Frauen) an der Studie teil. Die Ergebnisse konnten eine hohe Effektivität der im Rahmen des Netzwerkes angebotenen DBT nachweisen (Stiglmayr et al. 2015). Nur noch $23 \%$ der eingeschlossenen Patient_innen erfüllten nach einem Jahr DBT noch die Kriterien einer BPS. Die Anzahl an nicht-suizidalen Selbstverletzungen konnte signifikant reduziert werden. Innerhalb der Studienzeit gab es einen Suizidversuch, aber keinen Suizid. Die durchschnittlichen stationären Behandlungstage reduzierten sich von 51 Tage auf 7 Tage. Die Ergebnisse sind mit denen der publizierten RCTs vergleichbar (siehe Überblick in Bohus 2019). Eine Zusammenfassung der Ergebnisse findet sich in Tab. 1 ( $\mathrm{t} 0=$ Therapiebeginn; $\mathrm{t} 3=$ nach 12 Monaten).

Darüber hinaus konnte festgestellt werden, dass es bei Therapeut_innen, die an einem Konsultationsteam teilgenommen haben, zu signifikant weniger Therapieabbrüchen kam. Die Krankheitskosten konnten um ein Drittel reduziert werden; oder anders formuliert: für jeden ambulant investierten Euro konnten $2 €$ gespart werden (Wagner et al. 2013, 2014).

\section{Würdigung und Ausblick}

Zur Behandlung der Borderline-Persönlichkeitsstörung stehen mittlerweile mehrere gut evaluierte The-

Tab. 1 Median, Range, Mittelwerte $\left(M_{d}\right)$ und Standardabweichung $(S D)$ für paarweise Vergleiche t0-t3

\begin{tabular}{|c|c|c|c|c|c|c|c|c|c|}
\hline & \multicolumn{2}{|l|}{ to } & \multicolumn{3}{|l|}{ t3 } & \multicolumn{4}{|c|}{ Zeiteffekt } \\
\hline - & Median & Range & Median & Range & $n$ (Paare) & - & Wilcoxon U & $p$ & Effektgröße \\
\hline Anzahl Suizidversuche & 0,00 & $0-2$ & 0,00 & $0-1$ & 42 & - & - & $0,625^{\mathrm{a}}$ & - \\
\hline NSSV & 5,17 & $0-901$ & 1,00 & $0-174$ & 42 & - & $-3,03$ & 0,002 & 0,33 \\
\hline- & $M_{d}$ & $S D$ & $M_{d}$ & $S D$ & $n$ (Paare) & $t$ & $d f$ & $p$ & Effektgröße \\
\hline \multicolumn{10}{|l|}{ Stationäre Behandlung } \\
\hline Anzahl stationärer Aufenthalte & 1,13 & 1,41 & 0,32 & 0,89 & 47 & 3,85 & 46 & $<0,001$ & 0,56 \\
\hline Dauer (Tage) & 51,3 & 74,2 & 6,8 & 19,9 & 47 & 4,15 & 46 & $<0,001$ & 0,61 \\
\hline $\begin{array}{l}\text { Anzahl der DSM-IV TR BPD- } \\
\text { Kriterien }\end{array}$ & 6,4 & 1,2 & 3,2 & 1,9 & 31 & 8,85 & 30 & $<0,001$ & 1,59 \\
\hline \multicolumn{10}{|c|}{$\begin{array}{l}\text { Effektgrößen (Cohen's } d \text { ) basieren auf paarweise deskriptive Statistiken t0-t3 ( } p \text {-Werte paarweise Wilcoxon- and } t \text {-Tests) } \\
\text { Anzahl an Suizidversuchen, nicht-suizidales selbstverletzendes Verhalten (NSSV), Anzahl und Dauer stationärer Aufenthalte in den } 12 \text { Monaten vor to wird } \\
\text { vergleichen mit den } 12 \text { Monaten vor t3 } \\
\text { aMcNemar-Test aufgrund dichotomer Variablen (Suizidversuche Ja/Nein) }\end{array}$} \\
\hline
\end{tabular}


rapiemethoden zur Verfügung, die allesamt zeigen konnten, dass den Betroffenen effektiv geholfen werden kann. Auch hat sich gezeigt, dass die ambulante Psychotherapie einer stationären vorzuziehen ist nicht zuletzt aufgrund der deutlich geringeren Kosten und ihrer größeren Alltagsnähe. Trotz dieser enormen Fortschritte gerade in den letzten 20 Jahren, lehnen nach wie vor zahlreiche ambulante Psychotherapeut_innen die Behandlung ab. Dies hat ein beträchtliches Ausmaß an Leid für die Betroffenen aber auch deutlich höhere Kosten für das Gesundheitssystem zur Folge.

Die Dialektisch-Behaviorale Therapie (DBT) ist die Methode mit den meisten Wirksamkeitsnachweisen und findet seit den 1990iger Jahren zunehmende Verbreitung und zwar sowohl im vollstationären wie auch teilstationären Rahmen, in komplementären Einrichtungen sowie in der ambulanten Versorgung. Die DBT zeichnet sich durch ihre gute Anwendbarkeit im Rahmen unterschiedlichster Settings bei verschiedenen Störungsgruppen aus - beispielsweise findet die DBT auch zur Behandlung von Kindern- und Jugendlichen mit einer Affektregulationsstörung Anwendung. Da die DBT ein Teambasiertes therapeutischen Vorgehen darstellt, haben sich in den letzten Jahren immer häufiger Netzwerke gegründet, die eine Zusammenarbeit über mehrere Institutionen hinweg ermöglicht. Evaluierungsstudien aus Darmstadt und Berlin konnten die große Effektivität solcher Netzwerke zeigen (Friedrich et al. 2003; Stiglmayr et al. 2015). Zusätzlich zu ihrem Beitrag für eine verbesserte Versorgungslandschaft für Patient_innen mit einer BPS, wird immer wieder berichtet, dass die Zusammenarbeit in Netzwerken den Therapeut_innen Spaß und Zufriedenheit bereitet.

Es ist aus diesem Grund wünschenswert, dass solche Netzwerke eine weitere Verbreitung finden; sie sind kostengünstig, mit nur vergleichsweise wenig Aufwand verbunden - und sorgen dafür, dass man in Zusammenarbeit mit Kolleg_innen ein höheres Ausmaß an Arbeitszufriedenheit erlebt.

Interessenkonflikt C. Stiglmayr gibt an, dass kein Interessenkonflikt besteht.

Open Access Dieser Artikel wird unter der Creative Commons Namensnennung 4.0 International Lizenz veröffentlicht, welche die Nutzung, Vervielfältigung, Bearbeitung, Verbreitung und Wiedergabe in jeglichem Medium und Format erlaubt, sofern Sie den/die ursprünglichen Autor(en) und die Quelle ordnungsgemäß nennen, einen Link zur Creative Commons Lizenz beifügen und angeben, ob Änderungen vorgenommen wurden.

Die in diesem Artikel enthaltenen Bilder und sonstiges Drittmaterial unterliegen ebenfalls der genannten Creative Commons Lizenz, sofern sich aus der Abbildungslegende nichts anderes ergibt. Sofern das betreffende Material nicht unter der genannten Creative Commons Lizenz steht und die betreffende Handlung nicht nach gesetzlichen Vorschriften erlaubt ist, ist für die oben aufgeführten Weiterverwendungen des Materials die Einwilligung des jeweiligen Rechteinhabers einzuholen.

Weitere Details zur Lizenz entnehmen Sie bitte der Lizenzinformation auf http://creativecommons.org/licenses/by/4. $0 /$ deed.de.

\section{Literatur}

\section{Verwendete Literatur}

American Psychiatric Association (2003). Diagnostisches und Statistisches Manual Psychischer Störungen - Textrevision - DSM-IV-TR. Göttingen: Hogrefe. P. Falkai \& H.-U. Wittchen, deutscheAusgabe

Bender, D., Dolan, R., Skodol, A., Sanislow, C., Dyck, I., McGlashan, T., Shea, M., Zanarini, M., Oldman, J., \& Gunderson, J. (2001). Treatment utilization by patients with personality disorders. American Journal of Psychiatry, 158, 295-302.

Bohus, M. (2019). Borderline-Störung (2. Aufl.). Göttingen: Hogrefe.

Bohus, M., \& Wolf, M. (2013). Interaktives Skills-Training für Borderline-Patienten (2. Aufl.). Stuttgart: Schattauer.

Coid, J., Yang, M., Tyrer, P., Roberts, A., \& Ullrich, S. (2006). Prevalence and correlates of personality disorder in Great Britain. British Journal of Psychiatry, 188, 423-431.

Deutsche Gesellschaft für Psychiatrie Psychotherapie und Nervenheilkunde (DGPPN) (2009). Leitlinien für Persönlichkeitsstörungen. Heidelberg: Steinkopff.

Friedrich, J., Gunia, H., \& Huppertz, M. (2003). Evaluation eines ambulanten Netzwerks für Dialektisch Behaviorale Therapie. Verhaltenstherapie \& Verhaltensmedizin, 24, 289-306.

Fydrich, T., Renneberg, B., Schmitz, B., \&Wittchen, H. U. (1997). Strukturiertes Klinisches Interview für DSM-IV. Achse II. Persönlichkeitsstörungen (SKID-II). Göttingen:Hogrefe.

Germer, C. (2012). Der achtsame Weg zur Selbstliebe. Freiburg: Arbor.

Jobst, A., Hörz, S., Birkhofer, A., Martius, P., \& Rentrop, M. (2009). Einstellung von Psychotherapeuten gegenüber der Behandlung von Patienten mit Borderline Persönlichkeitsstörung. Ppmp - Psychotherapie - Psychosomatik - Medizinische Psychologie. https://doi.org/10.1055/ s-0029-1220764.

Lenzenweger, M.F., Lane, M.C., Loranger, A.W., \& Kessler, R. C. (2007). DSM-IV personality disorders in the National Comorbidity Survey Replication. Biological Psychiatry, 62, 553-564.

Lieb, K., Linehan, M. M., Schmahl, C., Zanarini, M., \& Bohus, M. (2004). Borderline personality disorder. Lancet, 364, 453-461.

Linehan, M. M. (1996a). Dialektisch-Behaviorale Therapie der BPS. München: CIP.

Linehan, M. M. (1996b). Trainingsmanual der Dialektisch-Behavioralen Therapie der BPS. München: CIP.

Stiglmayr, C.E., Stecher-Mohr, J., Wagner, T., Meißner, J., Spretz, D., Steffens, C., Roepke, S., Fydrich, T., SalbachAndrae, H., Schulze, J., \& Renneberg, B. (2015). Effectiveness ofdialecticalbehavioral therapyin routine outpatient care: The Berlin Borderline Study. Borderline Personality Disorder and Emotion Dysregulation, 1, 20. https://doi. org/10.1186/2051-6673-1-20.

Wagner, T., Fydrich, T., Stiglmayr, C. E., Marschall, P., Salize, H.J., Renneberg, B., Fleßa, S., \& Roepke, S. (2014). Societal cost-of-illness in patients with borderline personality disorder one year before, during and after dialectical 
behavior therapy in routine outpatient care. Behaviour Research and Therapy, 61, 12-22.

Wagner, T., Roepke, S., Marschall, P., Stiglmayr, C. E., Renneberg, B., Gieb, D., Dambacher, C., Matthies, S., Salbach-Andrae, H., Fleßa, S., \& Fydrich, T. (2013). Krankheitskosten der Borderline Persönlichkeitsstörung aus gesellschaftlicherPerspektive. Zeitschrift für Klinische Psychologie und Psychotherapie, 42, 242-255.

Wittchen, H.-U., Wunderlich, S., Gruschwitz, S., \& Zaudig, M. (1997). SKID. Strukturiertes Klinisches Interview für DSMIV. Achse I. Göttingen: Hogrefe.

Zanarini, M. C., Frankenburg, F. R., Hennen, J., Reich, D. B., \& Silk, K. R. (2004). Axis I comorbidity in patients with bor- derline personality disorder: 6-year follow-up and prediction of time to remission. American Journal of Psychiatry, $161,2108-2114$.

\section{Weiterführende Literatur}

Stiglmayr, C., \& Gunia, H. (2017). Dialektisch-Behaviorale Therapie zur Behandlung der Borderline-Persönlichkeitsstörung. Göttingen: Hogrefe.

Hinweis des Verlags Der Verlag bleibt in Hinblick auf geografische Zuordnungen und Gebietsbezeichnungen in veröffentlichten Karten und Institutsadressen neutral. 